\title{
AMÉRICA América
}

\section{Théâtre et exil entre le Río de la Plata et le Pacifique (1835-1855)}

Teatro y exilio del Río de la Plata al Pacífico (1835-1855)

\section{Edward Blumenthal}

\section{OpenEdition}

\section{Journals}

Édition électronique

URL : http://journals.openedition.org/america/4101

DOI : 10.4000/america.410

ISSN : 2427-9048

Éditeur

Presses Sorbonne Nouvelle

Édition imprimée

Pagination : 61-72

ISBN : 978-2-37906-063-2

ISSN : 0982-9237

Ce document vous est offert par Bibliothèque Sainte-Barbe - Université Sorbonne Nouvelle Paris 3

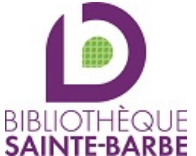

Référence électronique

Edward Blumenthal, "Théâtre et exil entre le Río de la Plata et le Pacifique (1835-1855) », América [En ligne], 54 | 2021, mis en ligne le 24 mars 2021, consulté le 29 mars 2021. URL : http:// journals.openedition.org/america/4101 ; DOI : https://doi.org/10.4000/america.4101 


\title{
Théâtre et exil entre le Río de la Plata et le Pacifique (1835-1855)
}

\author{
Teatro y exilio del Río de la Plata al Pacífico (1835-1855)
}

\begin{abstract}
Résumé
L'importance de la sphère publique habermassienne pour les jeunes romantiques du Río de la Plata et des pays voisins a reçu une attention particulière depuis quelques décennies. Cette nouvelle légitimation de la politique coïncidait avec des vagues importantes d'exil, notamment celle de la " jeune Argentine " qui s'opposa à la dictature du gouverneur de Buenos Aires, Juan Manuel de Rosas, et qui se dispersa sur les côtes du Pacifique, surtout dans les années 1840. L'objet de cette contribution est d'examiner comment le théâtre, déjà vu comme une pratique civilisatrice, se convertit en un lieu de sociabilité et d'expression politique, étroitement connecté au journalisme politique et au combat des émigrés. Des comédiens porteños en exil formèrent des compagnies qui voyagèrent le long des côtes du Pacifique, et un public d'émigrés les accueillit chaleureusement dans les théâtres et dans la presse, comme un symbole et une expression de leur situation politique.
\end{abstract}

Mots-clés : exil, théâtre, sphère publique, sociabilité, Amérique du Sud

\section{Resumen}

La importancia de la esfera pública habermasiana para los jóvenes románticos de Chile y el Río de la Plata ha recibido una atención creciente desde hace varias décadas. Esta nueva legitimación de la política coincidía con olas importantes de exilio, notablemente la de la "joven Argentina" que se opuso al dictador de Buenos Aires, Juan Manuel de Rosas, y se dispersó en las costas del Pacífico en las décadas de 1830 y 1840 . El objetivo de este artículo es examinar cómo el teatro, visto como una práctica civilizadora, se convirtió en un lugar de sociabilidad y expresión política, intimamente conectada al periodismo político y el combate de los emigrados. Actores porteños en exilio formaron compañías que viajaron por el Pacífico y un público de emigrados los acogió calurosamente en los teatros y en la prensa como un símbolo y una expresión de su situación política.

Palabras claves: exilio, teatro, esfera pública, sociabilidad, América del Sur 
C ET ARTICLE reprend un sujet classique de l'historiographie chilienne et argentine, à savoir la circulation du romantisme en Amérique du Sud avec l'émigration de la jeune génération de 1837, et les rapports entre ce groupe et la génération chilienne de 1842. L'objectif est de l'étudier à partir de deux perspectives liées : tout d'abord, les flux migratoires sur la côte du Pacifique, et deuxièmement la création de compagnies de théâtre dans ce cadre migratoire.

Le théâtre au Chili durant ces années s'est développé dans le contexte de la confluence de deux générations romantiques : celle de 1837 à Buenos Aires (Myers, 1998 ; Palti, 2009 ; Wasserman, 2008) et de celle de 1842 à Santiago (Subercaseaux, 1981). L'exil des membres de la génération romantique de 1837, qui tendait à se concentrer au Chili pendant cette décennie, a croisé le développement d'un mouvement parallèle à Santiago à partir de 1842. Ces générations romantiques participaient à la diffusion des idées de nationalité, c'est-à-dire la nation comprise dans son sens culturel et non seulement politique. Elles promouvaient la création d'une "littérature nationale ", une indépendance culturelle qui irait de pair avec la politique, mais dont les contours étaient toujours marqués par un fort américanisme, ce dernier renforcé par les interactions entre des personnes venant de différents pays américains. En Amérique du Sud, le romantisme s'identifiait plus particulièrement au socialisme utopique et plus généralement au libéralisme politique (Tarcus, 2016). Ces différents aspects du romantisme circulaient en Amérique du Sud mais avec une réception propre, qui générait des expressions politiques et culturelles particulières.

Durant cette décennie, des idées associées au romantisme étaient débattues dans la presse chilienne, et faisaient jaillir des polémiques souvent violentes (Jaksic, 2001 ; Stuven, 2000). C'est dans ce contexte que se développe aussi la critique de théâtre, marquée logiquement par les débats sur le romantisme, mais aussi par le contexte politique et la rencontre entre Chiliens et émigrés.

On analysera le théâtre comme faisant partie d'une sphère publique plus large. Cette notion, développée par Habermas (1991), est envisagée comme un espace intermédiaire entre la société et l'État, qui permet d'exercer une critique et un certain contrôle de ce dernier, indépendants de la sphère strictement politique du parlement. Bien que critiqué comme eurocentrique et élitiste, en particulier par l'idée d'une séparation catégorique entre sphères privée et publique (Fernández et Chassin, 2004 ; Guerra et Lempérière, 1998), le concept est utile dans la mesure où il permet de penser les phénomènes qui ne relèvent pas strictement des sphères politiques ou privées, et qui comprennent aussi des manifestations comme la presse n'appartenant pas forcément à un espace physique concret. II est lié aussi à la problématique de la sociabilité, dans la mesure où le théâtre était un lieu de sociabilité important pour les élites, plus public que les tertulias et salons encore nombreux à cette époque (González, 1999). Dans notre cas, étant donné la perception par les acteurs d'un « manque de public » - autant au sens figuré de l'opinion publique qu'au sens littéral d'un public physique et concret, pour les représentations théâtrales -, le théâtre se retrouve au carrefour de la sphère publique habermasienne et des espaces publics plus physiques.

On a aussi observé, dans une période antérieure, la façon dont les letrados utilisaient le théâtre comme outil politique, pour former et contrôler les populations. Il s'agissait de transmettre des enseignements moraux et civiques et combattre les divertissements méprisés de la plèbe. On pensait que le théâtre distrayait et occupait un peuple enclin à des mœurs dissolues, jouant un rôle important dans une "économie de l'attention " où il rivalisait avec d'autres distractions (Bowen Silva, 2016)'. En même temps, cette culture nationale ne l'est pas à proprement parler, à commencer par les réorientations culturelles. Si, par exemple, le théâtre argentin, compris comme expression d'une nation

1 Ce dernier inclut une synthèse de l'historiographie sur le théâtre dans le monde atlantique de l'époque. 
culturelle, est le produit du mélange du gauchesco et des compagnies d'immigrés italiens (Mandressi, 2008), cette expression n'était pas encore réalisée à l'époque étudiée ici. Les pièces théâtrales sont essentiellement européennes, et la nationalité culturelle en est encore à ses tout débuts.

\section{Les circulations dans le Pacifique}

Pour commencer, il convient d'ébaucher les espaces géographiques de circulation. D'un côté, nous avons l'espace Atlantique, avec des Européens, venant d'Espagne, d'Italie et de France (des comédiens, mais aussi des œuvres théâtrales) qui arrivaient parfois de Buenos Aires, mais aussi des Hispanoaméricains venant du littoral argentin (Baily et Míguez, 2003). De l'autre, un espace du Pacifique, s'étendant du Chili au sud jusqu'en Californie au nord, correspondant aux routes de cabotage de I'empire espagnol. Au Chili au XIXe siècle, on retrouve la convergence de ces deux grands espaces.

Des compagnies familiales, mal connues et très mal documentées, circulaient dans ces larges espaces. C'est ainsi que I'on retrouve au Chili la "Compagnie mexicaine " de Tiburcio López, venant du Mexique et de Cuba dans les années 1830 et 1840 (Pereira, $1974: 283$; Pradenas, $2002: 120$ ). Un autre exemple est Toribia Miranda, une "star " péruvienne qui a remporté un grand succès au Chili². Un article publié dans EI Mosaico, hebdomadaire qui publiait beaucoup de critiques de théâtre, indiquait comment les comédiens et autres métiers associés se recrutaient dans ces larges espaces, et les directeurs de compagnie étaient des entrepreneurs opérant à une échelle régionale avec une forte mobilité ("El teatro como especulación mercantil », El Mosaico, n 9, in Pereira, 1974 : 307-308).

La compagnie d'Hilarión Moreno et Dominga Montes de Oca, un couple marié arrivé au Chili en 1837, est un exemple de ces circulations. Moreno avait été en relation avec la politique unitaire à Buenos Aires où il occupa le poste de ministre des Relations extérieures sous Rivadavia, en 1825, avant de travailler dans la police en 1834. Bien qu'on ne le retrouve pas directement lié à la génération romantique de 1837, il étudie au Colegio de Ciencias Morales - fréquenté par l'élite porteña et il s'est exilé l'année de l'interdiction du Salon de 1837, où se réunissait la jeunesse romantique (Cutolo, 1968 ; Myers, 1998 ; Weinberg, 1958). Leur compagnie de théâtre devient rapidement une des plus réputées et des plus populaires, attirant non seulement les acteurs chiliens, mais aussi espagnols, outre la star péruvienne, Toribia Miranda. En 1839, avec le comédien espagnol Juan Peso, ils fondent le théâtre de l'Université sur le site où sera construit, quinze ans après, le Théâtre municipal (toujours existant). La construction a été financée par une souscription publique signée par la bonne société de Santiago (Pereira, 1974 : 200).

Cette compagnie cosmopolite accueillit aussi bon nombre d'émigrés de la Confédération argentine. Son directeur musical était le guitariste Marcos Ocampo, frère de Gabriel Ocampo, un émigré juriste naturalisé chilien qui allait être ensuite rédacteur du Code de commerce et membre de la Cour suprême du Chili. Parmi les grands noms de l'époque venant de Buenos Aires et travaillant dans la compagnie, on retrouve Máximo Jiménez, Victoriano Rendón, Carlos Fedriani et Juan Casacuberta (ibid. : 190-192). Même l'employé chargé de ranger les fauteuils (lunetas), de prendre les billets et de distribuer le journal était le domestique de l'émigré romantique Vicente Fidel López, Zacarias, qui l'avait accompagné en exil ("El teatro como especulación mercantil ", El Mosaico, n 9, in ibid. : 307308). Les mémoires du Chilien José Zapiola témoignent du rôle important joué par la compagnie de Moreno ${ }^{3}$. Zapiola précise que Juan Casacuberta, célèbre acteur porteño, était l'acteur le plus connu

2 "Lo que han sido el teatro y el público y lo que son ", El Mosaico, n 2, 21 juin 1846.

3 Pour le rôle de Zapiola dans la politique, voir Zapiola (1902). 
de la troupe de Moreno et Montes de 0ca et au Chili à cette époque. II attribue à ces trois porteños I'introduction du " théâtre moderne français " au Chili (Zapiola, $1974:$ 48).

Comme Moreno et Montes de Oca, Casacuberta était un émigré politique du rosisme. À la fin des années 1830, en raison du blocus français du port de Buenos Aires et des menaces d'une invasion d'émigrés à Montevideo, la répression sous la direction de Juan Lavalle se durcit à Buenos Aires, ce qui eut une conséquence directe sur le théâtre. Si auparavant, on avait l'obligation d'imprimer "Vive la fédération "sur les programmes, il était désormais exigé de déclamer des formules politiques au début des séances. La vie économique des théâtres était très instable et plusieurs furent contraints de fermer (Castagnino, 1989 : 369-371). Fin 1839, Casacuberta quitta Buenos Aires et alla rejoindre dans les provinces l'armée de Lavalle (ibid. : 385-386).

Comme la presse, le théâtre ressentit les effets de la répression de Rosas dans cette conjoncture critique, et fut une autre facette de l'unanimisme politique, terme utilisé pour décrire l'imposition souvent violente d'une seule opinion politique possible sous Rosas (Ternavasio, 1999). À l'inverse de la sphère politique au sens habermassien - de débat et médiation entre l'État et la société civile la presse, comme la scène, devint une émanation politique du pouvoir, sous la férule de la censure. Pareillement à la sphère publique qui à Buenos Aires se rétrécit et s'atrophie sous la pression de la dictature, l'espace public du théâtre subit les mêmes conséquences.

Casacuberta est arrivé au Chili en 1841 avec l'armée vaincue de Gregorio Aráoz de La Madrid, allié de Lavalle. Dès son arrivée, il a donné une série de spectacles organisée par la Commission argentine. Cette association d'émigrés implantée dans les principaux sites d'exil récoltait de l'argent pour les réfugiés et, plus largement, promouvait leur cause dans les journaux des pays d'accueil. Ces spectacles faisaient partie d'une campagne plus large où on trouve une souscription en faveur des émigrés, la collecte de vêtements et l'organisation d'un banquet pour fêter le général La Madrid - lui acheter un nouvel uniforme - et célébrer la cause anti-rosiste (Blumenthal, 2018). Ces spectacles présentaient Othello de Shakespeare et Marino Faliero de Donizetti (Quiroga Rosas à Sarmiento, 2 oct. 1841, in Sarmiento, $1850: 185)$. Les mémoires d'un émigré font mention de la présence des émigrés mais aussi des généraux de l'indépendance américaine issus de Lima, Santiago et Buenos Aires (Calzadilla, 1891 : 193-196). Le public était visiblement composé d'hommes provenant de différentes républiques du Pacifique, outre les émigrés de la Confédération argentine, ce qui souligne l'importance de Santiago dans les années 1840 en tant que lieu d'accueil.

Par la suite, Casacuberta rejoignit la compagnie de Moreno et devint une figure importante de la scène au Chili pendant toute la décennie, et pas seulement au Chili. En 1842, Moreno décida de partir à Copiapó : Santiago et Valparaiso se retrouvèrent sans compagnie théâtrale ("Teatro para Valparaíso ", El Mercurio, Valparaíso, 10 mai 1842, in Sarmiento, 1887, I : 260-263). II revint quelques mois après. Pour Sarmiento, ce retour représentait le salut du théâtre de la capitale et son port, qui agonisait sans le grand acteur porteño ("Representación del drama Mauricio o el médico i la huérfana ", El Progreso, Santiago, 10 déc. 1842, in Sarmiento, 1887, II : 64-66). En 1846, on retrouve Casacuberta à Lima où il a travaillé au Théâtre national. Par la suite, il s'est produit à Arequipa et Tacna, ville où il s'est marié ; le couple a eu un enfant en 1848 (Pereira, 1974 : 348). La compagnie de Moreno et Montes de Oca est revenue du Pérou en 1848 (ibid. : 335).

Casacuberta est mort, en 1849, d'une crise cardiaque après une interprétation de Les six grades du crime à Santiago. D'après le discours funèbre de Sarmiento, publié dans La Crónica, I'affiche du spectacle présentait le parcours du comédien. "Proscrit, errant, réchappé miraculeusement de sous la neige de la cordillère, je ne rêvais que du futur de ma patrie [...] presque aveugle dans ce pèle- 
rinage, j'ai trouvé de l'hospitalité et des mains bienfaitrices. Je me suis donc réconcilié, avec l'art " ("Casacuberta. Discurso pronunciado en su tumba ", in Sarmiento, $1899: 47$ ).

Si le conflit avec Rosas et l'exil correspondent à une interruption dans sa vie artistique, l'asile chilien a permis au comédien de remonter sur scène. Sa mort romantique, comme bien d'autres en exil, devint un fait de mémoire pour les émigrés, c'est ce qu'exprime le discours de Sarmiento. Cette nécrologie souligne aussi le rôle de ces circulations tant dans le théâtre que dans la politique. Casacuberta avait commencé sa carrière à Buenos Aires, " bouche par où [on] aspirait la civilisation européenne ". Comme Moreno ou Jiménez, il grandit dans le théâtre des années rivadaviennes. La politique les a repoussés vers les pays du Pacifique, où ils diffusaient la " civilisation " via le théâtre.

\section{Théâtre, romantisme et politique}

Déjà dans les années 1830, l'idée que le théâtre - et la critique de théâtre - était une force civilisatrice était répandue. Membre d'une "Commission de censure " nommée par l'État en 1830 pour promouvoir le théâtre face aux chinganas, guinguettes populaires, Andrés Bello regrettait que la " dignité et l'élévation artificielle de la tragédie exigent une perfection qui nous est inaccessible, dans l'état actuel de notre théâtre ". En même temps, il dut défendre le théâtre face aux attaques des prêtres et du milieu catholique conservateur. Ce faisant, il défendait la liberté romantique, pour trouver des expressions littéraires américaines (Pradenas, 2002 : 117-119). Bello a publié Staël, Chateaubriand, Hugo et Byron et il a traduit Dumas (Subercaseaux, $1981: 32$ ).

Le théâtre avait aussi une place importante au sein des préoccupations culturelles des jeunes romantiques des générations de 37 et 42, qui ont dédié une partie de leurs écrits à la critique théâtrale. José Victorino Lastarria, dont le discours a lancé la génération de 1842, a soutenu le théâtre dans les mêmes termes que Bello, même s'il s'est présenté postérieurement en opposition au conservatisme de son aîné (Jaksic, 2001 : 163-166). Dans son discours d'inauguration de la Société littéraire (1842), Lastarria s'est livré à une critique du passé colonial et a insisté sur la nécessité de créer une littérature américaine, mentionnant le poids de l'Espagne dans les livres scientifiques, la religion et le théâtre (Lastarria, 1885 : 106). Andrés Bello, pourtant ouvert au courant littéraire du romantisme, se méfiait de l'usage qu'en faisaient les jeunes romantiques d'un côté ou de l'autre de la cordillère, ce qui engendra une série de polémiques sur des questions littéraires (Jaksic, 2001 ; Stuven, 2000).

Ces débats incorporaient aussi les critiques du théâtre. Le théâtre était, pour Lastarria, un des terrains de lutte contre la culture espagnole (Lastarria, 1885 : 141). Il critiquait le théâtre des années 1830 pour s'être focalisé essentiellement sur "les émotions du sentiment " sans se préoccuper des "problèmes sociaux " (ibid. : 26). Cette critique est révélatrice du libéralisme social de l'avant-1848, propre à Lastarria, mais plus largement elle est aussi la façon dont le romantisme sud-américain s'exprimait en termes de socialisme utopique, sans toutefois taxer Lastarria d'un socialisme quelconque.

Lastarria souligne comment " le théâtre, les tertulias et les promenades " prenaient part à l'ouverture politique et intellectuelle des années 1840 (ibid. : 85). On est encore loin de la primauté de l'espace public comme lieu de débat; bien au contraire, on reste encore dans le cadre de l'espace privé des salons qui ont émergé ver la fin de l'ancien régime, analysés par François Xavier Guerra (1993). Cependant, on voit se profiler le théâtre comme un espace de débat véritablement public, promouvant la liberté et la régénération. Lastarria a présenté le théâtre comme un des trois " grands centres " du mouvement littéraire avec la Société littéraire et l'éducation publique (Lastarria, 1885 : 180).

Le théâtre était au cœur des préoccupations intellectuelles, en partie parce qu'il était considéré comme un élément de "civilisation " en mesure d'incorporer les classes populaires et de développer 
le "progrès ". En effet, les polémiques sur le romantisme ont commencé par des critiques de théâtre. Vicente Fidel López écrit un long article sur le romantisme où il souligne l'importance du théâtre comme " rempart " de la " civilisation du siècle " et comme " un des moyens par lequel la liberté moderne a démontré le pouvoir des idées ". II lie aussi l'état du théâtre au Chili - "nous n'avons pas de théâtre " - au besoin des élites de préserver leur pouvoir et leur position sociale ("Clasicismo y romanticismo ", Revista de Valparaíso, n 4, mai 1842, in Pinilla, 1943 : 14-15). Les réactions n'ont pas tardé, et l'hiver (juillet-août) 1842 s'échauffa en polémique autour du sujet.

Néanmoins, cette polémique se focalisait plus sur les structures politiques et sociales au Chili, et moins sur le romantisme à proprement parler. Parmi les opposants aux implications sociales explicites des propos de López et Sarmiento, qui se moquaient des gallicismes vides de sens et du " socialitisme " des émigrés, se trouvent quelques jeunes poètes romantiques, pourtant de tendance plus conservatrice, associés à la Société littéraire de Lastarria, comme José Joaquín Vallejo (Jotabeche) ou Salvador Sanfuentes (José Joaquín Vallejo, "Carta de Jotabeche a un amigo en Santiago ", El Mercurio, 23 juillet 1842, in Pinilla, 1943 : 40). Plus qu'une critique de théâtre, c'était un commentaire sur la société chilienne, perçue par beaucoup de jeunes romantiques comme conservatrice et fermée (Stuven, 2000 : 208-213).

Cela peut se voir aussi dans les critiques de Sarmiento des excès du romantisme, particulièrement une attitude gothique vide de critères littéraires ("La Nona sangrienta ", 29 août 1841. Sarmiento, 1887, I : 107-111, in Stuven, 2000 : 202-204). Si l'importance de cette révolution romantique était bien réelle pour Sarmiento, le conflit entre " classiques et romantiques " faisait partie du passé. Le romantisme était " une véritable insurrection littéraire " qui a " détruit les anciennes barrières " sans rien construire. C'est le "socialisme " qui lui succéda comme moyen de "réhabiliter le peuple, le mulâtre et tous ceux qui souffrent " ("Continúa el examen del artículo romanticismo ", El Mercurio, 28 juillet 1842, in Pinilla, $1943: 103)$. Ce sont l'organisation politique et sociale des sociétés américaines, et les limites de la démocratie qui faisaient l'objet du débat (Stuven, $2000: 211$ ).

Dans ces polémiques, on retrouve aussi un certain positionnement des émigrés au Chili comme "Argentins et romantiques ". Dans une lettre à un ami, López expliquait comment la polémique a façonné leur image au Chili. López était connu dans la société, "mais toujours comme Argentin, excitant une antipathie de nationalité ${ }^{4}$. Leur connaissance des auteurs romantiques et socialistes français n'était pas étrangère à cette réputation. López se moquait de ceux qui considéraient le romantisme comme " extravagant ", " absurde ", une " folie barbare qui n'a rien produit ": " donc toute l'Europe qui les a regardés comme des grands hommes est folle; donc le centre de la civilisation est au Chili et le centre du retard et de la folie est en Europe " (" Consideraciones sobre el romanticismo - III ", Gaceta del Comercio, 2 août 1842, in Pinilla, 1943 : 55). Cette même logique peut se discerner dans la façon dont Sarmiento mit fin à la polémique sur le langage en plagiant le romantique espagnol Mariano José de Larra pour affirmer que l'Espagne n'était en rien un modèle littéraire. Face à la critique de ses propos, il dévoila que les paroles du célèbre Espagnol avaient été copiées textuellement et fit ainsi taire ses détracteurs (Lastarria, $1885: 129)$.

Les polémiques sur le romantisme dépassaient largement le mouvement littéraire. Les " Argentins et romantiques " n'hésitaient pas à se moquer des excès du romantisme, alors que leurs rivaux, tels Bello ou Jotabeche, étaient également touchés par un certain style romantique. Les débats étaient l'enjeu de rivalités personnelles et politiques, mais cela menait à exacerber les différences de nationalité.

4 V. F. López à F. Frías, Santiago, 8 sept 1842, Revista biblioteca nacional, XXIV (58), 496. "Argentin et romantique ", p. 494. 


\section{Le théâtre et la civilisation}

L'idée du théâtre comme force civilisatrice trouvait peut-être son expression la plus énergique dans les critiques de Sarmiento sur le "retard du théâtre " au Chili ("El atraso del teatro ", El Mercurio, 7 juillet 1841, in Sarmiento, 1887, II : 72-75). II condamne l'utilisation d'un théâtre par un cirque équestre mais célèbre les rénovations annoncées à l'occasion de l'arrivée du comédien émigré Jiménez. II critique la posture des élites qui déprécient le métier de comédien, ce qui explique que les " mozos " (comédiens) soient quasiment analphabètes. Le théâtre est au " centre de la civilisation " en raison de ses composants : littérature, poésie, philosophie. La " poésie nationale " étant inexistante, il faut donc l'emprunter à l'Espagne et à la France, dont le défaut est d'être "étranger[s] "à " nos coutumes américaines ". II dénigrait aussi le théâtre de Valparaiso, "la première ville mercantile du Pacifique " qui avait " la superficie d'une arène de combat de coqs ". II en rend responsables les autorités publiques qui n'ont pas investi dans un théâtre digne de ce nom, et demande l'intervention de l'État pour la construction d'un théâtre national. Le théâtre était défaillant à l'image d'autres services basiques municipaux comme l'éclairage public et le pavage des routes ("Teatro para Valparaíso ", EI Mercurio, 10 mai 1842, in Sarmiento, 1887, I : 261). Pour Calzadilla, ce sont ces considérations qui avaient amené la création de la compagnie de Moreno et Montes de Oca (1891 : 194).

Le théâtre, pour Sarmiento, est plus qu'un " simple divertissement public ", comme les cirques équestres ; c'est surtout un "élément de culture " (El teatro como elemento de cultura ", El Mercurio, 20 juin 1842, in Sarmiento, 1887, I :271-275). S'il concède que le théâtre ne peut être " une expression de notre littérature " - car les Américains n'ont en ont pas - il est néanmoins, à ses yeux, un lieu privilégié qui accueille les idées européennes pouvant servir de modèle. Le "théâtre moderne " - et il pense ici à la France et à l'Espagne - "représente les besoins sociaux de l'époque " (ibid. : 272). II cherche à " détruire toute préoccupation de classe, toute tyrannie qu'elle soit publique ou domestique, et à élever à sa place la liberté individuelle des deux sexes ". C'est une façon d'avancer les idées républicaines qu'il soutient, mais aussi de promouvoir la culture aux " extrémités lointaines de I'Amérique ", où arrivent des acteurs comme "Casacuberta et Fedriani, comme Jiménez et Rendón " (ibid. : 273). Le théâtre est une façon de créer le peuple, d'élever la culture pour combattre les vices comme l'alcoolisme et le jeu ${ }^{5}$. Le romantique espagnol Larra, en particulier, est une référence, parce que " nous sommes une deuxième, troisième ou quatrième édition de l'Espagne [...] Ses vices sont les mêmes dont nous souffrons, fils d'une telle mère " (Las obras de Larra ", 31 août 1841, in ibid. : 111-114).

Le théâtre poursuivait un objectif social, dans le sillage des nouvelles doctrines du socialisme. C'est ainsi que dans une critique de la pièce Le Mulâtre, d'Alexandre Dumas, Sarmiento affiche sa sympathie pour le personnage qui doit lutter contre les préjugés de la société aristocratique pour obtenir la main d'une comtesse. Tout en reconnaissant que le public chilien ne va pas apprécier le mariage de ses filles à des hommes d'origine sociale douteuse, il défend la pièce, et plus généralement le théâtre, contre ses détracteurs qui la jugent comme une menace aux coutumes et à la moralité (" El Mulato. Drama de Alejandro Dumas ", El Mercurio, 7 juillet 1842, in ibid. : 279-282). Dans un article polémique déjà cité, Sarmiento était encore plus explicite : c'est la "nouvelle école, l'école socialiste " qui allait démontrer que "le mulâtre a plus de génie, de talent, de virtuosité, de magnanimité que le puissant, noble, corrompu, stupide " "Continúa el examen del romanticismo ". El Mercurio, 28 juillet 1842, in Pinilla, 1943 : 99). Ces idées socialistes permirent aussi de souligner les différences entre une Europe monarchique et une Amérique républicaine. II jugea que le récit de la corruption des cours

5 Cet article est cité textuellement avec approbation par Lastarria (1885: 185). 
royales, dans Le Roi s'amuse, d'Hugo, joué par Fedriani, Jiménez et Miranda, ne pourrait avoir lieu dans l'Amérique républicaine, car ce continent ne connaissait pas les "monstruosités " répandues dans les cours européennes ("El Rei se divierte. Drama de Victor Hugo ", in Sarmiento, 1887, II : 70-75). Certaines scènes ont apparemment choqué sa pudeur.

D'autres polémiques qui se sont développées tout au long de la décennie ont repris ces enjeux et souligné la place du théâtre dans ces débats. El Mosaico - hebdomadaire, apparu en 1846, qui accordait une grande place au théâtre - s'inquiétait de la réputation théâtrale et littéraire des Chiliens à l'étranger, en particulier au Pérou où les journaux circulaient, à la suite d'une série d'articles dans El Progreso intitulée "Estudios teatrales ", qui critiquait le théâtre chilien. L'auteur de l'article dans El Mosaico se consolait en arguant que l'auteur de l'article de El Progreso (Carlos Tejedor) était un étranger et regrettait que le journal rival ne mesure pas les avancées littéraires chiliennes ("Correo semanal ", El Mosaico, Santiago, n 6, 19 juillet $1846: 7-8$ ). C'est sans doute Pérez Rosales qui a écrit la chronique dans El Mosaico (Pérez Rosales, 1860 : 200-204).

Cependant, présenter le théâtre - et plus largement, la littérature - comme objet de discorde entre Chiliens et Argentins est exagéré. El Mosaico a reconnu la place des émigrés dans le théâtre chilien et se voulait un soutien du théâtre : "le théâtre n'est pas plus que le public et les périodiques ". $E$ I Mosaico rejoignait Sarmiento et Tejedor, soulignant le rôle du théâtre comme " une action civilisatrice pour toutes les classes de la société " et un élément du progrès ("La crítica teatral ", El Mosaico (Santiago), n 8, 2 août 1846 : 5), et critiquant le manque de théâtres et qu' « on ne représente jamais deux fois de suite une même pièce " pour des raisons économiques, le public le traitant comme une répétition ennuyeuse ("La crítica teatral ", El Mosaico (Santiago), n 6, 19 juillet 1846, 6-7).

Le théâtre chilien, si décrié par les émigrés de la Confédération, incluait déjà une forte présence étrangère. En effet, les critiques de théâtre visaient justement la compagnie de Moreno et Montes de $0 c a$, analysée dans la première partie. Cette opposition entre Chiliens et étrangers trouvait sa logique lorsqu'il s'agissait de tenter de définir des littératures " nationales ", et répondait aussi aux rivalités personnelles et aux styles polémiques de l'époque. Il y avait un intérêt à présenter ses ennemis comme des étrangers, et de la même façon pour des émigrés de se présenter comme "Argentins et romantiques ", ce qui signalait qu'ils avaient un accès privilégié aux savoirs européens. Dans ces polémiques - sur le théâtre et autres - le romantisme englobait des différences politiques plus importantes sur la vitesse des changements sociaux et l'extension de la démocratie, mais aussi sur la place des émigrés dans la société d'accueil.

\section{Théâtre, nationalités romantiques et littérature " américaine "}

Malgré le désir unanime de créer une littérature et un théâtre " nationaux ", la grande majorité des pièces produites étaient des traductions d'œuvres européennes, en particulier des romantiques. Shakespeare (Othello et Roméo et Juliette, entre autres) était populaire, et Casacuberta le représentait régulièrement, ainsi d'ailleurs que des Italiens, Donizetti en particulier. Souvent, les drames de Shakespeare étaient interprétés sous forme d'opérette italienne ("Romeo i Julieta. Ópera de Bellini ", El Progreso, 23 et 27 avril 1842, in Sarmiento, 1887, II : 181-186). Vicente Fidel López a traduit Une tache de sang de Julien de Mallian et Auguste-Louis-Désiré Boulé, qui a été représentée aussi par Casacuberta ("Una mancha de sangre ", El Progreso, 17 nov. 1842, in ibid. : 45-47). On appréciait des traductions de Victor Hugo ("Ánjelo ", El Progreso, 22 juillet 1843, in ibid. : 164-167; et aussi Ángel Tirano de Padua. "Las funciones teatrales del 18 de setiembre en Santiago ", 24 sept. 1841, in ibid. : 142-145) ainsi que d'autres pièces romantiques comme El Espía sin saberlo, qui a lieu pendant le 
premier consulat de Napoléon, et était populaire en raison de la prestation de Casacuberta ("El Espia sin saberlo. Una de tantas ", El Progreso, 26 août 1843, in Sarmiento, 1887, II : 170-173). López se plaignait de ne gagner pour ses traductions que l'équivalent de " 15 jours de dépenses courantes ${ }^{6}$ ".

Même si elles sont très minoritaires, les œuvres originales américaines sont intéressantes car elles reprennent des idées romantiques sur la nécessité de créer une littérature nationale. Bien avant la vague romantique, José Joaquín de Mora a composé la pièce El marido ambicioso à l'occasion de la promulgation de la Constitution de 1828. Mora était un émigré espagnol ayant été en exil successivement dans plusieurs pays d'Amérique du Sud. II a travaillé pour le gouvernement de Rivadavia à Buenos Aires, avant de passer au Chili en 1828 sous le président Francisco Pinto, mais il a été arrêté en 1831 et banni au Pérou (Iglesias, 2017). La pièce de Mora, écrite au Chili en 1828 pour fêter la constitution promulguée cette même année, a été reprise par la compagnie de Moreno et Montes de Oca en 1840 dans un clin d'œil à l'opposition chilienne pour qui la Constitution de 1828 était une référence (Pereira, $1974:$ :207-208).

Carlos Bello (fils d'Andrés) composa une pièce, Los Amores del poeta, dont l'inspiration romantique est claire. Elle a remporté un grand succès auprès du public à Santiago, et a reçu les éloges de El Mercurio (sous la plume du Néogranadien García del Río selon Lastarria) et de El Semanario. Cependant, Lastarria critiquait le fait que l'action se passe en France et non au Chili (Lastarria, 1885 : 184-187). Sarmiento émit des critiques similaires, demandant à quoi bon déplacer l'action sur un " sol étranger " quand l'Amérique avait aussi des "fleurs à cueillir, même si un peu sauvages " ("Los Amores del Poeta. Drama de Carlos Bello ", El Progreso, $1^{\text {er }}$ sept. 1842, in Sarmiento, 1887, 1 : 353-358).

Un autre Espagnol, Rafael Minvielle, a composé une pièce originale intitulée Ernesto, sur un officier espagnol qui abandonne les régiments du roi pour lutter au côté des Américains dans la révolution de l'indépendance, puis revient en Espagne où il n'est pas reconnu par les siens (Minvielle, Rafael, Ernesto, in Peña, 1912 : 73-130). Lastarria fait des éloges du sujet politique, et célèbre l'accueil d'une pièce américaine sur la scène chilienne, mais regrette le manque de perspective sociale (Lastarria, 1885 : 189-192). Sarmiento, lui aussi, a célébré une " composition contemporaine et nationale ", et il ne voulait pas " éteindre la flamme qui commence à prendre au sein de nos jeunes " avec des critiques trop sévères ("Ernesto. Drama de don Rafael Minvielle \#, El Progreso, 15 fév. 1843, in Sarmiento, 1887, II : 107-108).

Malgré cela, il caractérise l'auteur comme "Espagnol d'origine, bien qu'il soit, par sa longue résidence et par ses sympathies nouvelles et très cordiales, Américain et Chilien " (ibid. : 109). Sarmiento désapprouve le dénouement où Ernesto meurt en regrettant son engagement au service de la liberté américaine. II juge toutefois la pièce meilleure que celle de Bello et la compte parmi le "peu [d'œuvres] qui sont d'intérêt national et américain " (ibid. : 112). À l'opposé de sa critique de la pièce de Bello, Sarmiento pense qu'il aurait été " pédant " de localiser l'action en Amérique (et de substituer Napoléon par "O'Higgins, Carrera ou San Martín »), car la société américaine était " encore peu dramatique; trop simple dans ses relations". Si un drame veut "s'appuyer sur nos coutumes, qu'on le suppose possible ou vraisemblable dans notre société, il faut qu'il soit aussi simple et dénué qu'elle » (ibid.).

Ces pièces démontrent les tensions au sein du théâtre et de la littérature " nationale ". La recherche romantique des traits caractéristiques de la nationalité reposait en fait sur un mélange cosmopolite d'écrivains et de comédiens. Tout comme pour la compagnie de Moreno, les œuvres jouées, quand

6 V. F. López à V. López, Santiago, 23 nov. 1842 (AGN-López, Leg. 2364, nº 3960). 
elles étaient originales, montraient une ambiguïté fondamentale : elles sont plus américanistes que chiliennes. Théâtre national et jus soli ne s'accordaient pas.

\section{Conclusion}

Nous avons vu que le marché théâtral sur la côte Pacifique s'intégrait dans des circulations plus larges. Ce marché très réduit entraînait les déplacements constants des compagnies et des comédiens. II aurait été trop difficile économiquement parlant de rester dans une ville où le public, trop peu nombreux, était réticent à voir les mêmes productions. Dans ces circulations on trouve des Européens (des Français, des Italiens et surtout des Espagnols) mais aussi des Américains (du Río de la Plata mais aussi du Mexique ou du Pérou). Ils formaient des compagnies cosmopolites et écrivaient des pièces dont le caractère " américain " voire " national " n'échappait pas au public.

Le théâtre romantique était associé à une certaine sensibilité idéologique, liée au républicanisme égalitaire voire au socialisme pré-marxiste, mais était aussi intimement attaché à la question de l'exil et aux déplacements politiques. II visait entre autres à la création d'expressions culturelles nationales et se concevait comme un moyen idéal pour toucher un public plus large. Le théâtre, véritable extension de la presse, intégrait les débats de la sphère publique habermasienne. C'était aussi un espace physique qui accueillait le public désireux de participer à ces débats.

Néanmoins, à travers ces circulations - articulées à celle des journaux et livres qui n'a pas été directement abordée ici -, on a pu constater qu'au milieu du XIX siècle, les sphères publiques étaient - comme les sociétés - très peu nationalisées. C'est-à-dire que ce que l'on " consommait » à Santiago du Chili, dans la presse, le théâtre et bien d'autres domaines culturels, circulait aussi dans les autres centres dans l'aire du Pacifique et même au-delà. Ce qui était considéré comme national ou américain avait souvent des origines plus lointaines.

L'opposition entre Amérique et Europe en termes de "nationalité " et "littérature nationale " s'alimentait de ce fait. Pour les acteurs, il fallait civiliser (et se civiliser) au travers de la littérature, du théâtre, des journaux et des comportements venant d'Europe. Ces emprunts leur permettaient de créer une culture américaine et nationale. Vu le nombre d'émigrés politiques impliqués dans ces domaines, il est naturel qu'ils s'expriment souvent dans des termes toujours américanistes, même si des " rivalités de nationalité " entre Américains apparaissent.

Cette sphère avait pourtant tendance à se nationaliser tout au long du XIX siècle. Les théâtres étaient fréquentés par un public, de plus en plus compris dans un sens national, en l'occurrence chilien, et les circulations analysées ici jouaient un rôle dans cette nationalisation des sphères publiques. Les critiques théâtrales de la part d'u étrangers " provoquaient des levées de boucliers de la part des critiques chiliens, qui les voyaient comme des intrus étrangers et s'interrogeaient sur leur légitimité à participer à ces débats. Le théâtre, même cosmopolite, incluant non seulement des Chiliens mais aussi des Argentins, des Espagnols et des Italiens, qui jouaient des pièces françaises, s'affirmait de plus en plus comme chilien.

\section{Bibliographie}

BAlLY, Samuel L., et MíguEZ, Eduardo José, 2003. Mass Migration to Modern Latin America, Lanham (Maryland), Rowman et Littlefield.

BLUMENTHAL, Edward, 2018, " Exilio, guerra y política transnacional: las comisiones argentinas en la política internacional americana ", Anuario IEHS, 33 (2), p. 145-167. 
BOWEN SILVA, Martín, 2016, "Distraer y gobernar: teatro y diversiones públicas en Santiago de Chile durante la era de las revoluciones (1780-1836) ", Revista Historia, 49-I, p. 27-56.

CalzAdILLA, Santiago, 1891, Las Beldades de mi tiempo, Buenos Aires, Jacobo Peuser.

CAStAgnino, Raúl Héctor, 1989, El Teatro en Buenos Aires durante la época de Rosas, Buenos Aires, Academia Argentina de Letras.

CUTOLO, Vicente Osvaldo, 1968-1985, Nuevo diccionario biográfico argentino (1750-1930), 7 vol., Buenos Aires, Editorial Elche.

FERNÁNDEZ, Javier, et CHASSIN, Joëlle (dir.), 2004, L'Avènement de l'opinion publique. Europe et Amérique, XVIII'-XIXe siècles, Paris, L'Harmattan.

GonzÁlez BeRnaldo De Quirós, Pilar, 1999, Civilité et politique aux origines de la nation argentine. Les sociabilités à Buenos Aires 1823-1862, Paris, Publications de la Sorbonne.

GUERRA, François-Xavier, 1993, Modernidad e independencias: ensayos sobre las revoluciones hispánicas. Sección de Obras de historia, Madrid, Mapfre.

GUERRA, François Xavier, et LEMPÉRIĖRE, Annick (dir.), 1998, Los Espacio públicos en Iberoamérica. Ambigüedades y problemas. Siglos XVIII-XX, México, Fondo de Cultura Económica.

HABERMAS, Jürgen, 1991, The Structural Transformation of the Public Sphere: An Inquiry into a Category of Bourgeois Society, Cambridge (Mass.), The MIT Press.

IGLESIAS RogerS, Graciela, 2017, "José Joaquín de Mora in Chile: From Neo-Europe to the 'Beocia Americana' ", Bulletin of Latin American Research, 36 (3), p. 326-39.

JAKSIC, Ivan, 2001, Andrés Bello: la pasión por el orden, Santiago, Editorial Universitaria.

LASTARRIA, José Victorino, 1885, Recuerdos literarios, 2a ed., Santiago, M. Servat.

MANDRESSI, Rafael, 2008, "Du mélange des genres : le cirque "criollo" et le théâtre dans les pays du Río de la Plata (XIXXXe siècles) ॥, Communications, 83 (2), p. 211, https://doi.org/10.3917/commu.083.0211.

MORENo, Hilarión María, et MONTES DE OCA, Dominga, 1850, Colejio infantil para niños i niñas, calle de Duarte, acera del Dr. Nathaniel, Santiago, Impr. de Julio Belin.

MYERS, Jorge, 1998, "La Revolución en las ideas: La generación romántica de 1837 en la cultura y en la política argentinas ", Nueva Historia Argentina, tomo 3, Revolución, República, Confederación (1806-52), editado por Noemí Goldman, 3, p. 381-445, Buenos Aires, Editorial Sudamericana.

PALTI, Elías, 2009, El momento romántico, Buenos Aires, EUDEBA.

PEÑA, Nicolás, 1912, Teatro dramático nacional, vol. 1, Santiago, Impr. Barcelona.

PEREIRA SALAS, Eugenio, 1974, Historia del teatro en Chile: desde sus orígenes hasta la muerte de Juan Casacuberta, 1849. Santiago, Ediciones de la Universidad de Chile.

PÉREZ RoSALES, Vicente, 1860, Recuerdos del pasado (1814-1860), Santiago, Zig-Zag.

PINILLA, Norberto (dir.), 1943, La polémica del romanticismo en 1842: V.F. López, D.F. Sarmiento, S. Sanfuentes, Buenos Aires, Américalee.

PRADENAS, Luis, 2002, Le Théâtre au Chili - XVle-XXe, Paris, L'Harmattan.

SARMIENTO, Domingo Faustino, 1850, Recuerdos de Provincia, Santiago, Julio Belin y Cia.

—. 1887, Obras de D. F. Sarmiento, vol. 1-2, Santiago, Impr. Gutenberg.

—. 1899, Obras de D. F. Sarmiento, vol. 22, Buenos Aires, Mariano Moreno.

SobreVILla PereA, Natalia, 2011, The Caudillo of the Andes: Andrés de Santa Cruz, Cambridge, Cambridge University Press, New Approaches to the Americas.

STUVEN, Ana Maria, 2000, La seducción de un orden: las élites y la construcción de Chile en las polémicas culturales y politicas del siglo XIX, Santiago, Ediciones Universidad Católica de Chile.

SUBERCASEAUX, Bernardo, 1981, Cultura y sociedad liberal en el siglo XIX: Lastarria, ideología y literatura, Colección Bello, Santiago, Aconcagua.

TARCUS, Horacio, 2016, El socialismo romántico en el Río de la Plata (1837-1852), Buenos Aires, Fondo de Cultura Económica. 
TeRnAVASIO, M. 1999 " Hacia un régimen de unanimidad. Política y elecciones en Buenos Aires, 1828-1850 ", en SABATO, Hilda (dir.), Ciudadanía política y formación de las naciones. Perspectivas históricas de América Latina, México, Fondo de Cultura Económica, p. 119-141.

WASSERMAN, Fabio, 2008, Entre Clio y la Polis: Conocimiento histórico y representaciones del pasado en el Río de La Plata (1830-1860), Buenos Aires, Teseo.

WeInBERG, Félix, 1958, El Salón Literario, Buenos Aires, Hachette.

W00D, James A. 2011, The Society of Equality: Popular Republicanism and Democracy in Santiago de Chile, 1818-1851, University of New Mexico Press.

ZAPIOLA, José, 1974, Recuerdos de treinta años : 1810-1840, Santiago, Zig-Zag.

__ 1902, La sociedad de la lgualdad i sus enemigos, por José Zapiola, Santiago, G. E. Miranda. 